\title{
Geographical smoothing effects on wind power output variation in Japan
}

\author{
T Enomoto $^{\mathrm{a}}$, T Ikegami*a $^{\mathrm{a}}, \mathrm{C}$ T Urabe $^{\mathrm{b}}, \mathrm{T} \mathrm{Saitou}^{\mathrm{b}}, \mathrm{K}$ Ogimoto $^{\mathrm{b}}$ \\ ${ }^{a}$ Tokyo University of Agriculture and Technology, 2-24-16 Naka-cho, Koganei-shi, Tokyo 184-8588, Japan \\ ${ }^{b}$ Institute of Industrial Science, the University of Tokyo, 4-6-1 Komaba Meguro-ku, Tokyo 153-8505, Japan
}

\begin{abstract}
There is a growing concern that the supply and demand operations of electric power systems will become more difficult with a large number of renewable energy sources whose outputs depend on the weather variations. To investigate the countermeasures that are required to stabilize the outputs of the power systems, it is important to quantitatively evaluate the wind power output fluctuations and variations corresponding to the supply-demand balancing controls, such as turbine governor control, load frequency control, and economic-load dispatching control (EDC). With an increasing number of wind power generators being installed, these fluctuations and variations with respect to the rated output capacity will decrease because of the geographical smoothing effects, i.e., the output fluctuations and variations of multiple wind turbines will mutually cancel one another. In this study, we quantitatively evaluate the geographical smoothing effects using the actual high time-resolution wind power output data of several wind farms that is recorded in the supervisory control and data acquisition systems of the Tohoku and Kyushu power systems in Japan. Further, we evaluate the smoothing effects pertaining to the introduced wind power using various approximation curves, and it is observed that the exponential parts of the approximation curves depend on the region and cycles. Using these exponential parts, we observe that perfect smoothing effects were obtained during the shorter periods of cycles. The smoothing effects of the fluctuations in the EDC domain are observed to be small, especially in the Tohoku region.
\end{abstract}

Keywords: Wind power, smoothing effect, power fluctuation

\section{Introduction}

Toward sustainable low-carbon society, several countries are initiating the usage of renewable energy sources. Renewable energy is an environment-friendly power generation technology because it does not emit carbon dioxide during power generation. However, its output varies from moment to moment under the influence of the sun, clouds, and wind. Therefore, when these renewable energy systems are introduced on a large scale into various power systems, the supply and demand operations of various electric power systems are likely to become more difficult because of the aforementioned output fluctuations and variations.

When a number of wind power generation systems are installed, the fluctuations in the wind power output and rated output capacity will become small because the output fluctuations and variations of multiple wind turbines mutually cancel each other; this mutual cancellation is called the smoothing effect. Therefore, it is necessary to quantify these smoothing effects to estimate the future wind power output fluctuations and variations. A few studies have been conducted to investigate the influence of long-term variations on the smoothing effect [1]. However, to the best of our knowledge, no study has analyzed the short-term fluctuations that affect the normal supply-demand balancing operations in a power system. To investigate the countermeasures against output fluctuations and variations in power systems, it is

\footnotetext{
* Manuscript received January 10, 2018; revised January 28, 2018.

Corresponding author. Tel.:+81-42-388-7435; E-mail address: iket@cc.tuat.ac.jp

doi: $10.12720 /$ sgce.7.3.188-194
} 
important to quantitatively evaluate the future wind power output fluctuations and variations corresponding to the supply-demand balancing systems, such as turbine governor control (TGC), load frequency control (LFC), and economic-load dispatching control (EDC).

In this study, we have quantitatively evaluated the geographical smoothing effects using the actual high time-resolution wind power output data of several wind farms (WFs). These data were recorded by the supervisory control and data acquisition (SCADA) systems located in the Tohoku and Kyushu power systems in Japan. The details of the SCADA data that were used to conduct this study are described in Section 2.1. To associate the wind power generation output fluctuations with the time range of the frequency controls of the power system, high time-resolution SCADA data was divided into three fluctuations (corresponding to TGC, LFC, and EDC) and a long-term variation data by applying the moving average filter. Three frequency controls are described in Section 2.2, and the dividing methods are described in Section 2.3. It is necessary to cover most of the range of distribution of the fluctuations. Further, the shape of their distributions is observed to be different from the normal distribution. Therefore, we adopted the 0.1th and 99.9th percentile values for the distribution of fluctuations without using the standard deviation $(\sigma)$ or $3 \sigma$ as an evaluation index of the output fluctuations.

According to the central limit theorem, the standard deviation of the synthesized output fluctuations becomes $N^{0.5}$ times the standard deviation of individual WFs when the output fluctuations of the $N$ WFs are independent of one another. Thus, the installed capacity $(N)$ of the wind power generation decreases as a negative exponential function $\left(N^{-0.5}\right)$ because of the smoothing effects. However, if the wind power output fluctuations are not completely independent, the smoothing effects are observed to be weaker than those of the completely independent fluctuations. Therefore, in this study, we analyze the fluctuations by which the output variations vary with respect to the rated output capacity, corresponding to the installed capacity. The details of the analysis methods are described in Section 3. In Section 4, we describe the results of the analysis of the Tohoku and Kyushu electric power systems in Japan.

\section{High Time Resolution Wind Power Output Data}

\subsection{Used data set}

In this study, we used SCADA data of each WF in Tohoku and Kyushu where wind power generation systems were installed in Japan. The time resolution of the Tohoku data was $10 \mathrm{~s}$, whereas the time resolution of Kyushu data was $2.5 \mathrm{~s}$. The data are collected from 0:00 on April 1, 2012 to 24:00 on March 31, 2013. Additionally, we used a per-unit system (p.u.) for the power outputs and fluctuations. The outputs value (p.u.) are calculated by dividing the actual outputs value by the capacity of the WFs. The arrangement of the WFs and details of the data are depicted in Table 1 and Figure 1.

Table 1. Characteristics of SCADA data of the two power systems

\begin{tabular}{lccc}
\hline & Time resolution [s] & The number of WFs & Total capacity [MW] \\
\hline Tohoku & 10 & 17 & 439 \\
Kyushu & 2.5 & 16 & 307 \\
\hline & & & \\
\hline
\end{tabular}

Fig. 1. Location of the wind farms in the Tohoku and Kyushu regions 


\subsection{Frequency Controls in Power Systems}

To supply stable electric power, the electric power company controls the output of each power station, such as thermal and hydro power, in response to the fluctuating electric power demand. Therefore, supply and demand are observed to be always consistent with each other. Further, we also observe that demand is controlled by different methods, such as TGC, LFC, EDC, depending on the cycle band.

In TGC, a speed governor is used, which is a device that adjusts the steam and water quantity in order to keep the rotational speed of the generator constant. It is an operation that automatically adjusts the output of the generator by following the change of system frequency by placing it in a state of response. The TGC supervises over the adjustments in fluctuations of a short cycle ranging from several tens of seconds to several minutes.

In LFC, the central load dispatching center (CLDC) detects the deviation of the frequency of the power system and fluctuation of the interconnection line current flow. Further, the CLDC sends the control signal to the power plant and generates the output of the generator such as thermal and hydro power. LFC maintains the system frequency to be constant by automatically controlling it. Further, LFC controls the adjustment of the fluctuation of the cycle from several minutes to $20 \mathrm{~min}$ of the fluctuation cycle of demand. In Japan, the amount of electric power that is adjustable by the LFC (i.e., the LFC adjustment power) is generally observed to be approximately $5 \%$ of the system capacity.

In the EDC, the CLDC estimates the power allocation of each thermal power/hydro power generator based on the power demand forecast and change in demand. Simultaneously, the allocation of power to each generator is determined to be the most economical. Although EDC can adjust the amount of electric power with respect to LFC, it requires time to start up the thermal power generator. Therefore, EDC is responsible for adjusting the fluctuations of the cycles by several tens of minutes or more. The schematic for the sharing of each control is depicted in Figures 2 and 3[2][3][4].

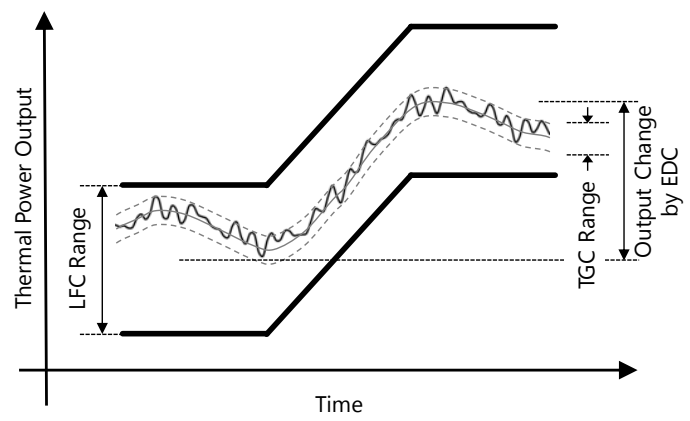

Fig. 2. The thermal power output controls [2]

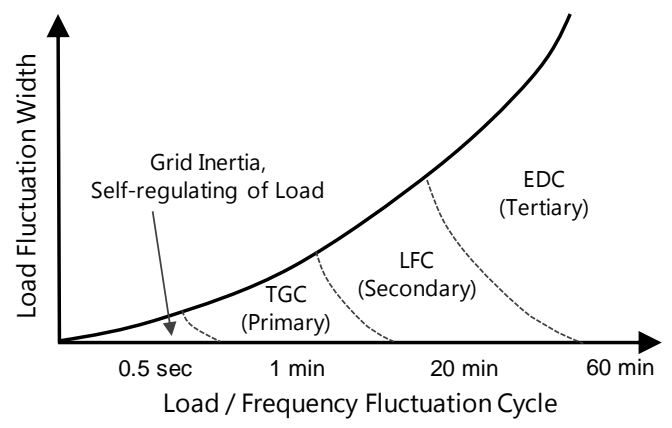

Fig. 3. Assigned target domains for each type of frequency control [3] 


\subsection{Definitions of Output Fluctuations for Power Systems Operations [5]}

As mentioned in Section 2.2, the actual supply/demand balancing adopts a different method for each cycle. Therefore, in this analysis, we divided the output into three variations using the centred moving average (CMA). From the original output data $\left(p_{t}\right)$, we calculated the CMA for $1 \min \left(C M A_{t}^{(I m i n)}\right), 30 \mathrm{~min}$ $\left(C M A_{t}^{(30 \text { min })}\right)$, and $3 \mathrm{~h}\left(C M A_{t}^{(\text {(hour })}\right)$. The fluctuations that are followed by the TGC domain $\left(r_{t}^{(T G C)}\right)$ are considered to be the difference between the outputs and $C M A_{t}^{(I \min )}$ in equation (1). Further, the fluctuations of the LFC domain $\left(r_{t}^{(L F C)}\right)$ are considered to be the difference between the $C M A_{t}^{(I m i n)}$ and $C M A_{t}^{(30 m i n)}$, as depicted in equation (2), whereas the fluctuations that are followed by the EDC domain $\left(r_{t}^{(E D C)}\right)$ are considered to be the difference between the $C M A_{t}^{(30 m i n)}$ and $C M A_{t}^{(3 h o u r)}$, as depicted in equation (3). In these equations, $t$ represents the time step.

$$
\begin{aligned}
& r_{t}^{(T G C)}=p_{t}-C M A_{t}^{(1 \min )} \\
& r_{t}^{(L F C)}=C M A_{t}^{(1 \mathrm{~min})}-C M A_{t}^{(30 \mathrm{~min})} \\
& r_{t}^{(E D C)}=C M A_{t}^{(30 \mathrm{~min})}-C M A_{t}^{(3 \text { hour })}
\end{aligned}
$$

\section{Analysis Procedure}

\subsection{Central limit theorem}

It has been confirmed that the variation rate becomes smaller when the wind power generation outputs are added, which is called the smoothing effect. Theoretically, it is considered that the standard deviation of the total output becomes $N^{0.5}$ times the standard deviation of the individual output while adding $N$ outputs that are not correlated with one another [6][7]. For example, consider summing the independent $N$ outputs with a rated capacity of $1 \mathrm{~kW}$. The standard deviation of the total output $\left(\sigma_{\text {total }}\right)$ is depicted in equation (4). The standard deviation of each WF is observed to be $\sigma_{n}$.

$$
N \sigma_{\text {total }}=\sqrt{\sum_{n=1}^{N} \sigma_{n}{ }^{2}}
$$

For simplicity, let us assume that the probability distributions of the output fluctuations at each WF are the same, whereas the output fluctuations in real time are different from one another. Therefore, $\sigma_{\text {total }}$ is given as follows:

$$
\sigma_{\text {total }}=\frac{1}{N} \sqrt{N \sigma_{n}^{2}}=\frac{\sigma_{n}}{\sqrt{N}}=\sigma_{n} N^{-0.5}
$$

However, practically, the output fluctuations at each WF are not independent, and the rated capacity of each WF is not the same. In this situation, the absolute value of the exponent of $N$ would be smaller than that of equation (5) when adding positive correlated fluctuations, such as long-term fluctuations.

Therefore, we expressed the smoothing effect using the exponential function of the introduction capacity of wind power and evaluated the effect by comparing the exponent with -0.5 in this study.

\subsection{Percentile}

As depicted by Ikegami et al. [5], the actual WF output of the probability and normal distributions are observed to be different. Thus, the average $(3 \sigma)$, which is usually the range from which almost all the values of normal distribution are obtained, is observed to be different from the $3 \sigma$ of the actual wind power fluctuation distribution. Therefore, we used the 99.9th percentile as a representative value of variations in this study. As depicted in Figure 4, the 99.9th percentile is the value at the region that is located at $99.9 \%$ from the bottom of the distribution. Further, we obtained the 99.9 th percentile value by combining the fluctuations and expressing the smoothing effect by quantitatively analyzing how the value decreases. 


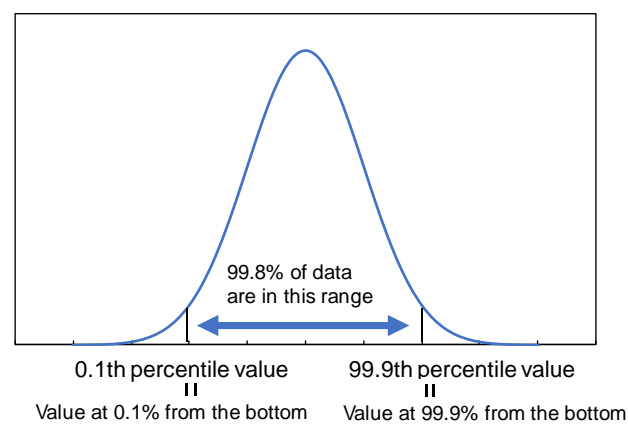

Fig. 4. The 0.1th and $99.9^{\text {th }}$ percentiles in the distribution

\subsection{Evaluation methods of smoothing effect}

In wind and solar power generation, many small-scale systems such as wind turbines and solar panels are distributed and installed over an extensive region. However, the weather conditions are not always identical in each region. Therefore, there are fluctuations in the amount of electricity that is generated by each generator. The fluctuations in the total amount of generated electricity are reduced by mutual cancelations (i.e., the smoothing effect). To effectively obtain the smoothing effect, it is necessary to distribute photovoltaic and wind power generation over an extensive region, depicting different weather conditions. However, we observe that not all fluctuations in the short cycle are canceled because of the smoothing effect. Fluctuations due to large variations such as weather also remain, and these increase with the introduction of wind and solar power generation. Further, it is expected that the problems pertaining to supply and demand adjustments due to long-time domain fluctuations, such as day and night and seasonal fluctuations, will become obvious as the introduction progresses.

In this study, the distribution of fluctuations of wind power output combined with multiple WFs in each region and cycle domain was obtained. The smoothing effect was expressed by decreasing the 99th percentile value of the distribution with an increase in total wind power capacity. We plotted all the combinations with the capacity on the $x$ axis and 99.9th percentile value on $y$ axis, as depicted in Figure 5(a). Further, we drew an approximate curve by exponential approximation $\left(y=a x^{b}\right)$. While drawing the approximate curves, the number of combinations was observed to be concentrated in the middle. Therefore, to reduce the bias of weight in each plot, the average value was estimated for every $10 \mathrm{MW}$ and an approximate curve of the average value was drawn using the least-square method, as depicted in Figure 5(b). Additionally, we obtained the duration curve of the output in each region to investigate the smoothing effect in the distribution of the output.

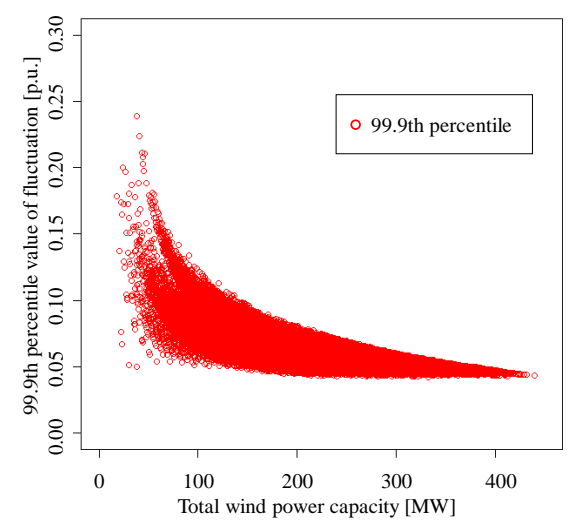

(a)

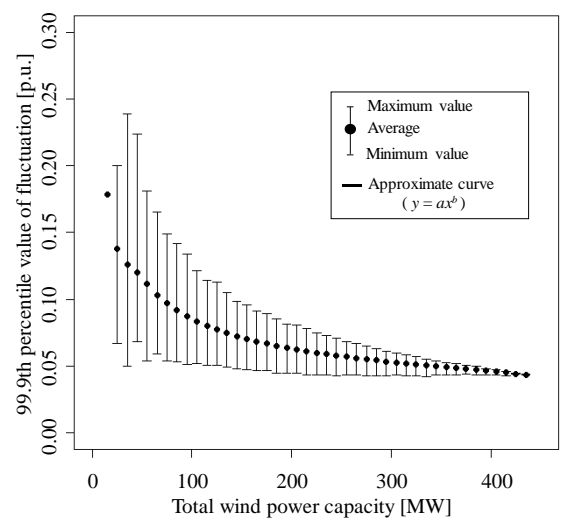

(b)

Fig. 5. (a) All the combinations with the capacity on the $\mathrm{x}$ axis and 99.9th percentile value on $\mathrm{y}$ axis and (b) approximation curve as a function of the total wind power capacity in the Tohoku region. 


\section{Results}

\subsection{Smoothing effect on the 99.9th percentile value of fluctuations}

The obtained approximation curves are depicted in Figures 6 (a) and (b) in the Tohoku and Kyushu regions in each cycle. As can be observed from these figures, the value of $b$ is observed to be approximately -0.5 in the TGC domain in both regions, and the smoothing effect is obtained well in the LFC domain. Additionally, it can be confirmed that the smoothing effect becomes difficult to obtain as the cycle becomes longer. From Figure 6 (a), the exponent of the approximation curve in Tohoku is observed to be -0.491 , which is very close to -0.5 . From this observation, we can assume that the variation in the TGC domain is independent and that a large smoothing effect is obtained. However, in the EDC domain, the exponent of the approximation curve is observed to be -0.233 , which depicts that it is difficult to obtain the required smoothing effect as compared to the shorter period. This could be caused due to their positive correlation with each other as the cycle becomes longer.

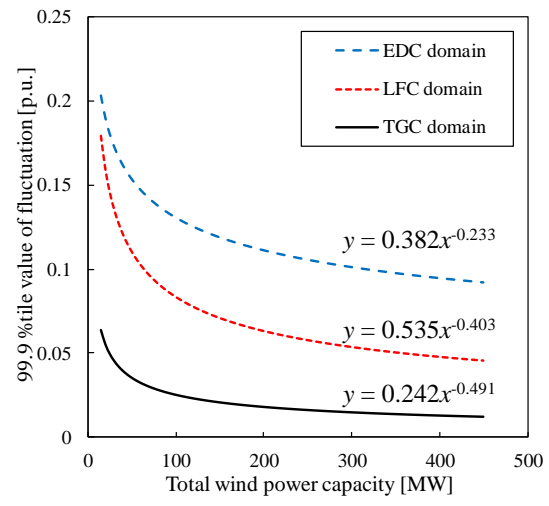

(a)

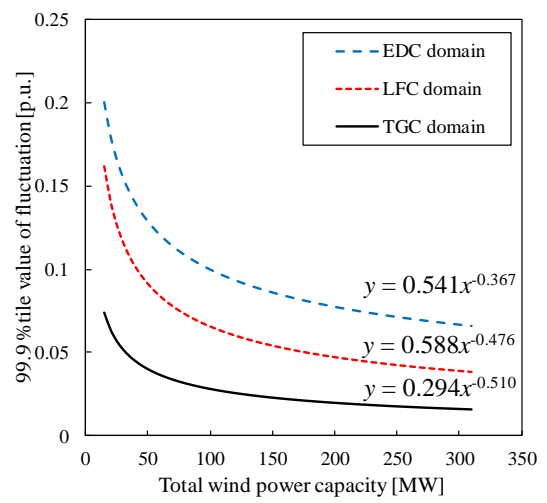

(b)

Fig. 6. The approximation curves: (a) the Tohoku region and (b) the Kyushu region.

\subsection{Duration curve}

The duration curve of the output in each region is depicted in Figures 7 (a) and (b). In these figures, it can be observed that the sum of the outputs (red line) passes through the middle of the whole lines and has a smaller inclination than each line of a single WF at the cumulative time from 1000 to $6000 \mathrm{~h}$. Further, in both the Tohoku and Kyushu regions, the output is observed to be approximately zero at about $1800 \mathrm{~h}$ for $1 \mathrm{WF}$, whereas the output instantaneously exceeds 1.0 per unit (p.u.) in some WFs. However, there is almost no time for the sum of the outputs to be zero. The maximum output value was observed to be approximately 0.9 p.u. in Tohoku and 0.8 p.u. in Kyushu. Thus, it was confirmed that the distribution of the output was entirely adjusted by the smoothing effect.

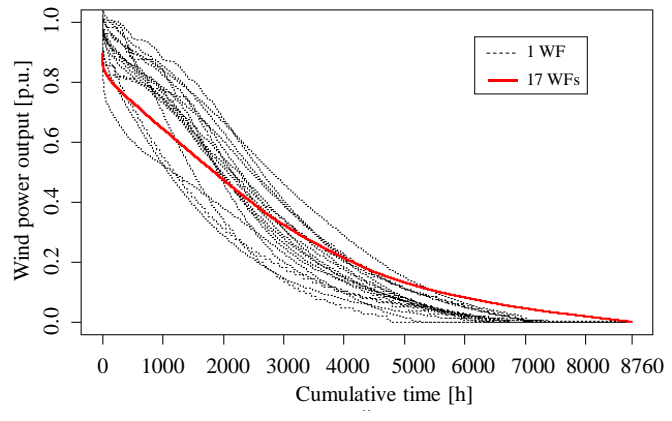

(a)

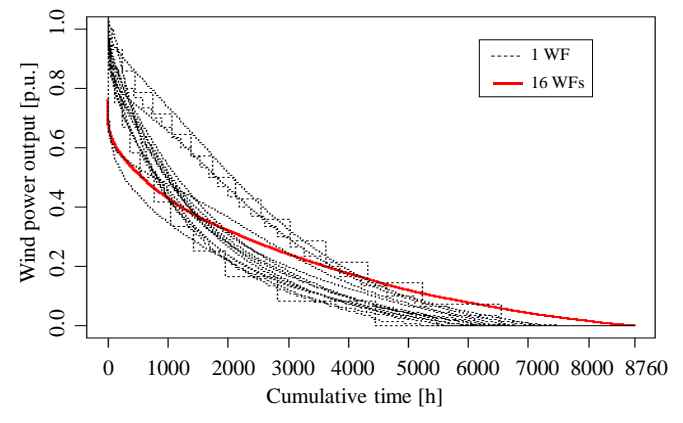

(b)

Fig. 7. The duration curves of wind power output in a year: (a) the Tohoku region and (b) the Kyushu region. 


\section{Conclusion}

In this research, we quantitatively evaluated the smoothing effect by analyzing the output data of WFs in the Tohoku and Kyushu regions where wind power generation is being introduced progressively. We estimated the distribution shapes of wind power output fluctuations for every combination of WFs on the three cycles by considering the controls in the actual electric power systems and by plotting the 99.9th percentile with regard to the wind power capacity. Further, we determined the approximation curves that were represented by the smoothing effects. It was observed that the exponential parts of the approximation curves were dependent on the region and cycles. Using these exponential parts of the approximation curves, we observed that perfect smoothing effects were obtained during the shorter periods of cycles, whereas the smoothing effects of the fluctuations in the EDC domain were observed to be small, especially in the Tohoku region.

Additionally, it was also confirmed that the slope of the duration curves become gentle and the frequency of extreme events near the maximum and minimum output decreased.

\section{Acknowledgements}

Wind data used in this work was collected through collaborative research by the University of Tokyo and Japan Wind Power Association, and provided by the Ogimoto Laboratory in the University of Tokyo. This work was partially supported by R\&D Project on Grid Integration of Variable Renewable Energy: Mitigation Technologies on Output Fluctuations of Renewable Energy Generations in Power Grid, commissioned by the New Energy and Industrial Technology Development Organization (NEDO).

\section{References}

[1] Buttler A, Dinkel F, Franz S and Spliethoff H. Variability of wind and solar power - An assessment of the current situation in the European Union based on the year 2014. Energy. 106 pp 147-161 (2016) https://doi.org/10.1016/j.energy.2016.03.041

[2] Investigating R\&D Committee of the Institute of Electrical Engineers in Japan Analysis and Operation Technologies for Power System Utilization IEEJ Technical Report, No. 1100, 2007 (in Japanese)

[3] Investigating R\&D Committee of the Institute of Electrical Engineers in Japan, Load Frequency Controls on a steady and emergency basis in Power Systems IEEJ Technical Report, No. 869, 2002 (in Japanese)

[4] A. Tani. Advances in Steam Turbines for Modern Power Plants, Chapter8: Steam turbine design for load-following capability and highly efficient partial operation, Woodhead Publishing; 2016.

[5] Ikegami T, Urabe C T, Saitou T and Ogimoto K. Numerical Definitions of Wind Power Output Fluctuations for Power Systems Operations. Renewable Energy. 115 pp 6-15 (2018) https://doi.org/10.1016/j.renene.2017.08.009

[6] Kato T, Kumazawa S, Honda N, Koaizawa M, Nishino S and Suzuoki Y. Development of Estimation Method of Spatial Average Irradiance Fluctuation Characteristics Considering Smoothing Effect around Observation Point. Electronics and Communications in Japan. 97(11) pp1-9 (2014) https://doi.org/10.1002/ecj.11595

[7] Kato T, Inoue T and Suzuoki Y. Estimation of Total Power Output Fluctuation of High Penetration Photovoltaic Power Generation System. 2011 IEEE Power and Energy Society General Meeting. pp1-9 https://doi.org/10.1109/PES.2011.6039560 